\title{
Imaging and Spectroscopy of Low-Dimensional Low-Z Materials by 20-300kV TEM
}

\author{
Ute Kaiser $^{1 *}$
}

1. Central Facility of Materials Science Electron Microscopy, Ulm University, Ulm, Germany.

* Corresponding author: ute.kaiser@uni-ulm.de

The field of low-dimensional (low-D) materials has advanced enormously since graphene's discovery in 2004 and the prospect for new applications of novel condensed matter systems is triggering new avenues to control, measure and understand their properties. As properties often are determined by single atoms, a quest for "seeing" single, even light atoms and to understand chemical bondings has triggered the development of a new type of transmission electron microscopes, the so-called SALVE microscope. It operates at electron accelerating voltages between $80 \mathrm{kV}$ and $20 \mathrm{kV}$ with sub-Angstroem resolution down to $40 \mathrm{kV}$ as well as energy-filtered imaging in very high resolution by correcting not only the geometrical aberrations of the objective lens but also its chromatic aberration in a large field of view in a single image. Here we will very briefly outline the instrumental advances [1-3]. The work proposed aims at combining and advancing (a) structure preparation routes, (b) high-resolution and energy-filtered TEM imaging and spatially- and momentum-resolved EELS methodology and (c) theoretical modelling to understand the intricate interplay between crystallographic and electronic structure.

The low-D and low-Z-number objects are truly 2D materials (graphene, hBN and carbon nitrides), metal clusters confined in single walled carbon nanotubes and few-layer 2D polymers composed of areal repeat units of 2D polymer crystals, which just very recently was successfully grown [4].

We report on the dynamics of Li-diffusion in-between graphene during lithiation and delithiation and show that even single Li atoms can be resolved after removing the contribution of graphene by Fourier filtering [5]. This approach does not work for the case of momentum-resolved EELS, as Coulomb interaction allow receiving the response from the full heterostructure only [6]. Graphene, hBN [7,8] and carbon nitrides [9] show interesting optical properties and collective phenomena; in these materials, quantum confinement effects lead to strongly bound excitons and first experiments will be presented. In the case of metal clusters confined in single-walled carbon nanotubes [10] we image the atomic-scale dynamics of a $\mathrm{Re}_{2}$ molecule adsorbed on a carbon lattice, which allows direct measurement of $\mathrm{Re}-\mathrm{Re}$ bond length for individual molecules that changes in discrete steps correlating with bond order from 1 to 4 , depending on orientation of $\mathrm{Re}_{2}$ molecule with respect to the carbon lattice (see Fig. 1a).

For the case of two-D polymers, we resolve the porphyrin and linker units with an image resolution of 2.5 Angstrom with low-dose 300kV HRTEM imaging [11]. In order to enhance the image contrast under low-dose condition (100 electrons/angstrom ${ }^{2}$ ), we optimized the phase contrast transfer function to extract the low spatial frequency information (see Figure 1b). In addition, we carried out electron diffraction tomography to determine the 3-dimentional unit cell dimensions and symmetry. Furthermore, we present work on optimization of imaging conditions with respect to total electron dose and specimen resolution in dependence on the electron acceleration voltage between $300 \mathrm{kV}$ and $30 \mathrm{kV}$ [12].

References:

[1] M Linck et al., Physical Review Letters 117 (2016), p. 076101. 
[2] F Börrnert and U Kaiser, Physical Review A 98 (2) (2018), p. 023861.

[3] www.salve-project.de

[4] R Dong, T Zhang and X Feng, Chem. Rev. 118 (2018), p. 6189.

[5] M Kühneet al., Nature 564 (2018), p. 234.

[6] MJ Mohnat al., Physical Review B 97 (2018), p. 235410.

[7] JC Meyer et al., Nano Letters 9 (2009), p. 2683.

[8] O Cretu et al., ACS Nano 8 (2014), p. 11950.

[9] G Algara-Silleret et al., Angewandte Chemie 53 (2014), p. 7450.

[10] K Caoet al., Nanoletters 18 (10) (2018), p. 6334.

[11] H Sahabudeen et al., Nature Communications 7 (2016), 13461.

[12] We acknowledge funding from the DFG and the MWK of the Federal state of Baden-Württemberg, Germany, in the frame of the SALVE project (www.salve-project.de.) as well as from the BadenWürttemberg Stiftung $\mathrm{GmbH}$ in the frame of the CleanTech program and the European Union in the frame of the Graphene Flagship.
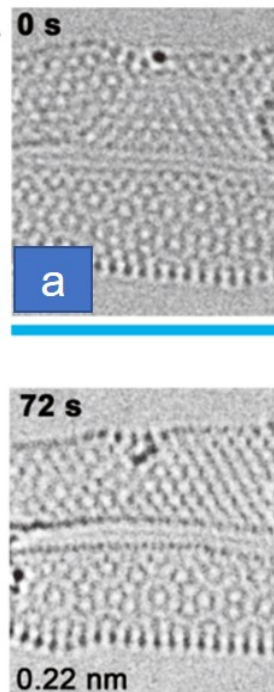

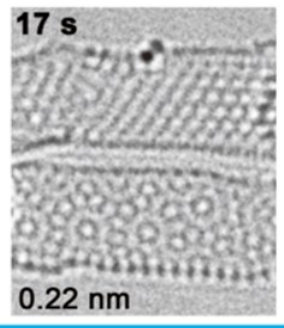

Lie down and rotate

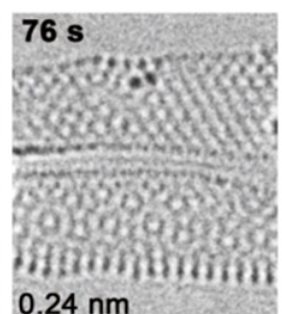

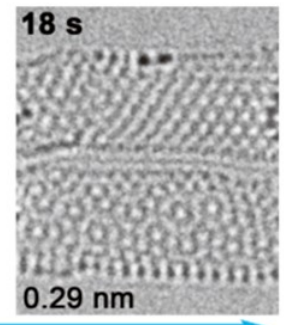

$0.29 \mathrm{~nm}$

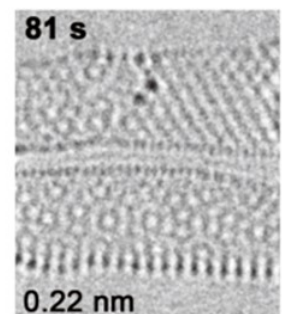

Walk on the outer surface

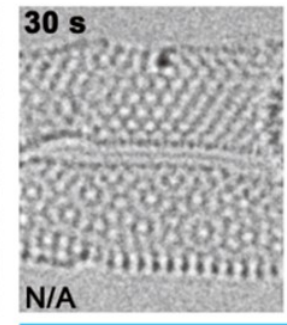

N/A

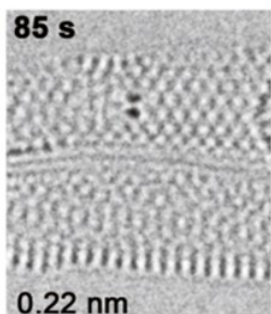

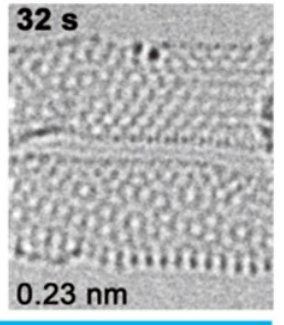

Lie down and stand

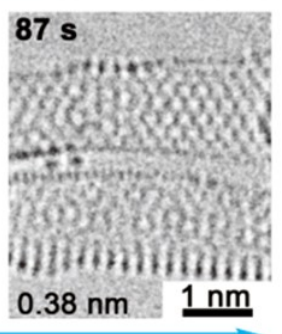

Slide into Van der Waals gap

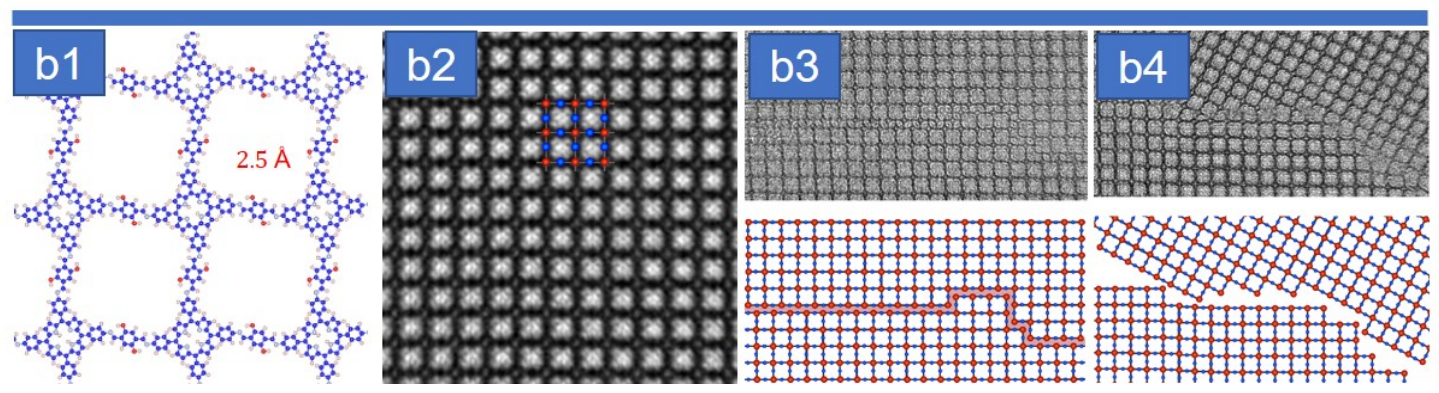

Figure 1. (a) $80 \mathrm{kV} \mathrm{Cc/Cs-corrected} \mathrm{HRTEM} \mathrm{time-series} \mathrm{images.} \mathrm{The} \mathrm{dirhenium} \mathrm{molecule} \mathrm{moves} \mathrm{at} \mathrm{a} \mathrm{defect} \mathrm{site}$ on SWNT, climbs out from the defect and then slides into a vdW gap between two parallel SWNTs. The corrected Re-Re bond length of each frame is presented, measurement error is $\pm 0.010 \mathrm{~nm}$ (b1) Atomic model of 2D polyimine synthesized at an air-water interface. (b2) 300kV Cs-corrected HRTEM image (denoised) of 2D polyimine under low-dose condition (total electron dose: c.a. $100 \mathrm{e}^{-} / \AA^{2}$ ) showing the square lattice of polyimine. The red and blue spheres represent porphyrin and linker units, respectively. (b3) HRTEM image (raw) and schematic of an antiphase domain boundary. (b4) HRTEM image (raw) and schematic of a high-angle grain boundary. 